\title{
Thoracoscopic sympathectomy for chronic ischaemic pain in the upper extremity
}

Samarasinghe $\mathrm{T}^{1}$, Galketiya $\mathrm{KB}^{1}$, Pinto $\mathrm{V}^{1}$

${ }^{\prime}$ Professorial surgical unit, Teaching Hospital Peradeniya

Ke y w ord s - Th or a c o s c o p y, Sympathectomy, Buerger's, Limb Ischaemia, Peripheral vascular disease

\section{Introduction}

Thrombangitisobliterans (Buerger's disease) is a non-atherosclerotic inflammatory disease which affects small \& medium sized vessels (1).The incidence of this condition variable in different countries from $5-50 \%$ of the diagnosed patients with PVD. Of these $1 / 4^{\text {th }}$ is described to be involving the upper limbs. More often, Buerger's disease is not amenable to surgical revascularization and when advanced,leads to debilitating disease culminatingin multiple extremity gangrene and amputations. However sympathectomy has been reported to alleviate pain and help in superficial ulcer healing (2).

We present a patient with Buerger's disease who was successfully treated by thoracoscopicsympathectomy for ischaemic pain of left hand.

\section{Case report}

A forty four year old male with a history of 25 pack years of smoking presented with dry gangrene of 2 digits of left hand \& debilitating rest pain. The onset of his symptoms was at the age of 34 years \& he has undergone right side below knee amputation 5 years ago. His CT angiogram confirmed Buerger's disease not amenable to revascularization. Patient was advised on smoking cessation \& pharmacological therapy was initiated but showed minimal response with regard to pain control after 6 months. Therefore thoracoscopicsympathectomy was discussed and consent obtained.

Patient was intubated with single lumenendotracheal tube and both lungs were ventilated. Patient was positioned in semi-prone position. Lung collapse was obtained with a capnothorax of $8 \mathrm{mmHg}$. Three ports (Figure 1) were used.

Sympathectomy from $2^{\text {nd }}$ to $4^{\text {th }}$ Thoracic ganglia was performed using monopolar diathermy hook. There was no measurable blood loss and duration of procedure was 20 minutes.Postoperative intercostal drainage was not used. The 2 gangrenous digits were amputated and wounds were kept open. They showed delayed but positive evidence of healing on follow-up

Patient was started on oral feeding and mobilized out of bed on same day. Objective pain assessment using visual analogue scale (VAS) showed a significant decline from $8 / 10$ to 3/10.Patient was discharged on D2 of surgery. Patient's pain remained at $3 / 10$ on VAS at 6 weeks review (Figure 2). 


\section{Discussion}

Afferent pain impulses from the extremities reach the CNS via both dorsal root ganglion and sympathetic fibres. Of these general visceral afferent fibres associated with transmission of ischaemic pain predominately follow sympathetic pathwaysthrough sympathetic ganglia and reach dorsal root ganglia via white rami communicans (3). Thus disruption of sympathetic chain causes immediate pain relief (4). Also sympathetic efferents innervate dermal capillary bed which once disrupted causes capillary dilatation leading to increased dermal blood flow which intern is believed to aid in wound healing (5).

\section{Conclusions}

Thoracoscopic techniques are preferable due to the improved visualization, markedly less surgical morbidity as well as faster recovery (6).

Thoracic sympathectomy is effective to relief ischaemic pain in Buergers disease. It can be effectively performed by thoracoscopy which allows early discharge from hospital.

Prof KB Galketiya,

Professorial unit,

Teaching hospital,

Peradeniya.

kbgalketiya@yahoo.com

Submitted by - May 2019

Accepted by - June 2019

\section{References}

1. Dargon PT, Landry GJ et al.Buerger'sdisease.AnnVasc Surg. 2012 Aug;26(6):871-80. doi: 10.1016/j.avsg.2011.11.005. Epub 2012 Jan 27.

2. Coveliers HM, Hoexum F, Nederhoed JH, Wisselink W, R a u werda JA. Thoracic sympathectomy for digital ischemia: a summary of evidence. J Vasc Surg. 2011 Jul;54(1):273-7. doi: $10.1016 /$ j.jvs.2011.01.069. Epub 2011 Jun 8

3. Stranding S, editor. $41^{\text {th }}$ ed. London: Churchill Livingstone; 2016. Autonomic nervous system. Gray's Anatomy: The Anatomical Basis of Clinical Practice; pp. 233-236 https://doi.org/10.1002/ca.22677

4. A. Matarazzo, V. Rosati-Tarulli, O. Sassi, A. Florio, M. Tatafiore, C. Molino. Possibilities at present for the application of lumbar sympathectomy in chronic occlusive arterial disease of the lower limbs. Minerva Cardioangiol, 50 (2002), pp. 363-369; PMID : 12147968 I S S N : $\begin{array}{llllllll}0 & 0 & 2 & 6 & -4725\end{array}$ https://www.minervamedica.it/en/jo u r n a l s / m i n e r v a cardioangiologica/article.php?cod= R05Y2002N04A0363\&acquista $=1$

5. Matsumoto Y, Ueyama T, Endo M, Sasaki H, Kasashima F, Abe Y, Kosugi I. Endoscopic thoracic sympathicotomy for Raynaud's phenomenon. J Vasc Surg. $2002 ; 36: 57-61$. do i : https://doi.org/10.1067/mva.2002.1 23330 
6. Hashmonai M, Cameron AEP, Licht $\mathrm{PB}$, Hensman C, Schick CH. Thoracic sympathectomy: a review of current indications. SurgEndosc 2016;30:1255-69. doi:10.1007/s00464-015-4353-0.

\section{Figures}

Figure 1. Patient positioning and port placement for thoracoscopicsympathectomy

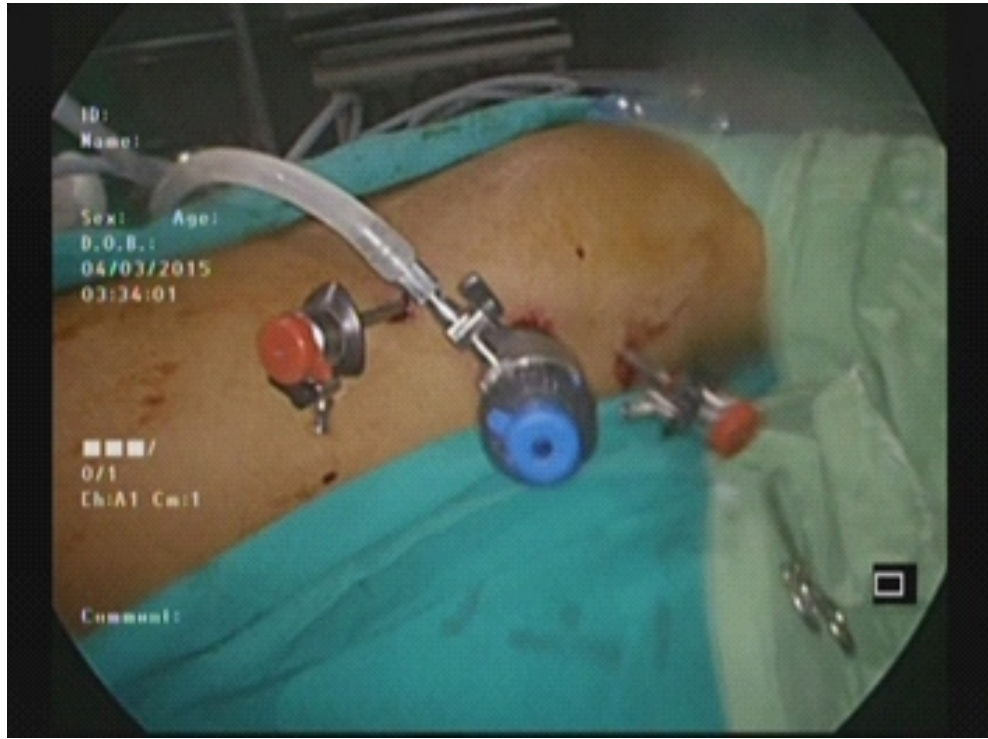

Figure 2. Thoracoscopicsympathectomy with monopolar hook

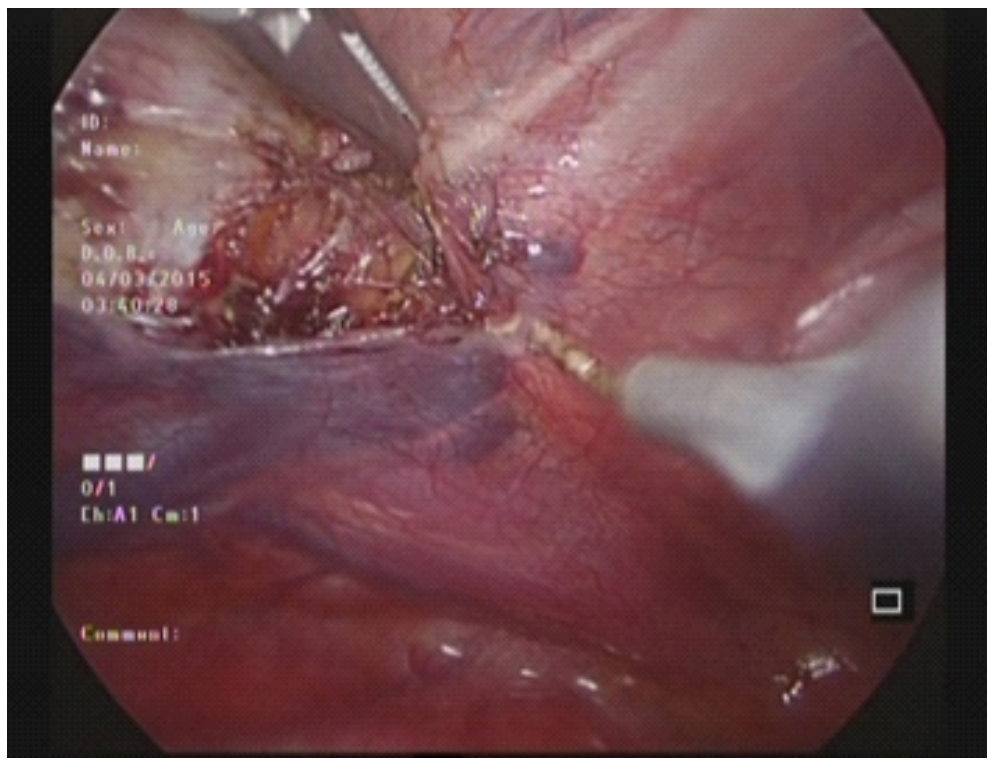

